\title{
Power Loss Models in Punched Non-Oriented Electrical Steel Rings
}

\author{
Nicholas Lewis ${ }^{1}$, Philip Anderson ${ }^{1}$, Jeremy Hall ${ }^{1}$, and Yi Gao ${ }^{2}$ \\ ${ }^{1}$ Wolfson Centre for Magnetics, Cardiff University, Cardiff CF24 3AA, U.K. \\ ${ }^{2}$ Research and Development, Tata Steel, Coventry CV4 7HP, U.K.
}

\begin{abstract}
The stresses and strains generated during punching of electrical steel laminations degenerate the magnetic properties in the region near the cut edge. The existing work has tended to focus on strips, with estimates of the damaged width ranging from less than 1 up to $10 \mathrm{~mm}$. In this paper, ring samples, which are more representative of motor laminations, are considered. Increasing the diameter of the samples and, therefore, increasing the proportion damaged by the punching allowed the creation of mathematical models to describe and analyze the power loss and estimate the extent of the damaged region. Five samples each measuring five laminates high with a constant outer diameter of $200 \mathrm{~mm}$ and various inner diameters $(150,160,170,180$, and 190 mm) were stamped from coils of $\mathrm{M250-35A}$ and M330-35A of a non-oriented electrical steel. A simple model was initially proposed, where power loss is proportional to the ratio of the undamaged to the total width and the square of the flux density passing through that region. An average width for the damaged region was calculated at $0.31 \mathrm{~mm}$ for the two materials.
\end{abstract}

Index Terms-Electrical steel, non-oriented (NO), power loss, punching.

\section{INTRODUCTION}

$\mathbf{T}$ HE increasing demand for more efficient motors and, therefore, for the optimal construction of electrical machines requires the designers to possess a detailed knowledge of the magnetic properties of the $0.2-1 \mathrm{~mm}$ thick nonoriented (NO) electrical steel laminations that are stacked together to produce the motor cores. These properties, including power loss and permeability that are measured by manufacturer tests on steel sheets postproduction, are different from those of the final laminations due to stresses and strains introduced by manufacturing processes, such as punching, welding, and shrink fitting, which cause a deterioration of the magnetic properties [1], [2]. The amount of deterioration depends on cutting per unit volume, cutting angle relative to the rolling direction, and the silicon content [3]. Punching and shearing of the laminations introduce plastic strains [4] and the creation of long-range residual stresses [5]. The plasticity causes a decrease in the permeability and an increase in the power loss localized in a narrow region close to the cut edge [6] with the heaviest impact around the key parts of the motor, such as the teeth. This local effect could have a large global impact. Plastic deformation is the result of movement of defects or irregularities in the crystal, known as dislocations. The presence of dislocations hinders the movement of domain walls, which increases the hysteresis loss.

Plastic deformation has been observed in [7] in NO SiFe $(2 \% \mathrm{Si}) 0.5 \mathrm{~mm}$ thick Epstein strips in a region up to $3 \mathrm{~mm}$ from the cut edge. Schmidt [8] identified a $0.35 \mathrm{~mm}$ wide area of edge hardening in $\mathrm{SiFe}(1 \% \mathrm{Si})$.

Estimates for the width of the damaged area on Epstein strips range from $1.5-2.5 \mathrm{~mm}$ by Senda et al. [4],

Manuscript received August 31, 2015; revised December 18, 2015 and January 12, 2016; accepted February 11, 2016. Date of publication March 8 , 2016; date of current version April 15, 2016. Corresponding author: N. Lewis (e-mail: lewisnj1@ @ardiff.ac.uk).

Color versions of one or more of the figures in this paper are available online at http://ieeexplore.ieee.org.

Digital Object Identifier 10.1109/TMAG.2016.2530304
$1.9 \mathrm{~mm}$ by Kuo et al. [9], 5-6 $\mathrm{mm}$ by Loisos and Moses [10], and up to $10 \mathrm{~mm}$ by Rygal et al. [3] for $0.5 \mathrm{~mm}$ thick NO SiFe. Nakata et al. [11] identified a $10 \mathrm{~mm}$ width in $0.35 \mathrm{~mm}$-thick NO SiFe. Gmyrek et al. [12] calculated the damaged width of NO SiFe $0.5 \mathrm{~mm}$ thick ring samples using an inverse algorithm to be 1.40 and $1.87 \mathrm{~mm}$ using analytical and finite element method approaches, respectively.

The magnetic properties of the laminations in electrical machines will be impacted by the stresses and strains generated in the manufacturing process. The understanding and modeling of the complexities of this problem are still an active area of research.

The purpose of this paper was to identify the effect that the cut edge due to punching had on the magnetic properties using ring samples manufactured using an industrial punch and die rather than a simulated process on straight samples. The rings simple geometry allowed for uniform magnetization compared with the more complex shapes of real stators.

This paper presents models describing the power loss due to punching, which can aid designers in the construction of efficient electrical machines involving more complicated punching patterns, for example, the teeth.

\section{METHOD}

The loss, apparent power, and permeability of ring laminates with various inner diameters (IDs): (150, 160, 170, 180, and $190 \mathrm{~mm}$ ) and constant outer diameter (OD) $200 \mathrm{~mm}$ stamped from coils of M250-35A and M330-35A of (NO) electrical steel were measured.

Stacks of each sample five rings high at each ID were tested in a computer controlled, ac magnetizing system developed at the Wolfson Centre [13], consisting of a desktop PC, National Instruments Data Acquisition, power amplifier, and isolation transformer. The 310 turn primary windings were wound on three formers, to reduce compressive stress and increase air cooling. As the flux density produced is inversely proportional to the number of primary turns and the frequency, the number 


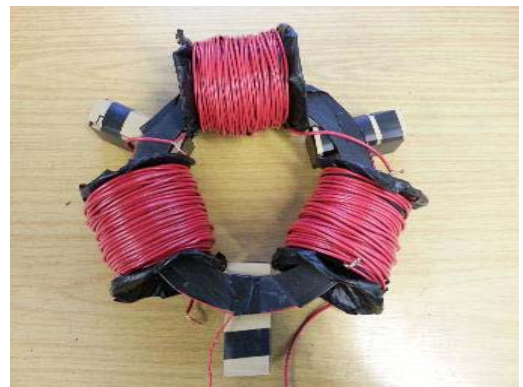

Fig. 1. Ring samples with primary windings on winding formers.

TABLE I

MAGNETIZATION CONDITIONS FOR RING SAMPLES

\begin{tabular}{cc}
\hline \hline Frequency & ${\text { Flux Density }(\mathrm{T})^{\mathrm{a}}}^{\mathrm{a}}$ \\
\hline $50 \mathrm{~Hz}$ & $1.0 \mathrm{~T} / 1.3 \mathrm{~T} / 1.5 \mathrm{~T} / 1.7 \mathrm{~T}$ \\
$100 \mathrm{~Hz}$ & $1.0 \mathrm{~T} / 1.3 \mathrm{~T} / 1.5 \mathrm{~T} / 1.7 \mathrm{~T}$ \\
$200 \mathrm{~Hz}$ & $1.0 \mathrm{~T} / 1.3 \mathrm{~T} / 1.5 \mathrm{~T} / 1.7 \mathrm{~T}$ \\
$400 \mathrm{~Hz}$ & $1.0 \mathrm{~T} / 1.3 \mathrm{~T} / 1.5 \mathrm{~T} / 1.7 \mathrm{~T}$ \\
$2.5 \mathrm{kHz}$ & $1.0 \mathrm{~T} / 1.3 \mathrm{~T}$ \\
$5 \mathrm{kHz}$ & $0.1 \mathrm{~T}-0.7 \mathrm{~T}^{*}$ \\
$10 \mathrm{kHz} y$ & $0.1 \mathrm{~T}-0.3 \mathrm{~T}^{*}$ \\
\hline
\end{tabular}

* or maximum flux density possible dependent on material properties.

\begin{tabular}{|c|c|c|}
\hline$\mu_{d}$ & $\mu_{\mathrm{u}}$ & $\mu_{\mathrm{d}}$ \\
\hline$=\Phi_{\mathrm{d}}$ & $\Phi_{\mathrm{u}}$ & $\Phi_{\mathrm{d}}$ \\
\hline$d / 2$ & & $\mathrm{~d} / 2$ \\
\hline
\end{tabular}

Fig. 2. Idealized slice of ring along the radial direction. With height $h$ and width $w=(\mathrm{OD}-\mathrm{ID}) / 2$.

of turns was successively reduced at higher frequencies. A four turn secondary winding was wound around each stack. The three winding formers are not in a direct contact with the ring sample which sits freely on the supporting blocks, so that no additional stress is applied. The ring is therefore balanced and stress free, as shown in Fig. 1.

Loss, permeability, apparent power, and ac $B-H$ loops were measured in all the sample packs at the range of frequencies and flux densities presented in Table I. Five different rings were measured with consistent results indicating a consistency in the material properties and the stamping process.

Consider a thin slice of the ring cut along the radial direction with width $=(\mathrm{OD}-\mathrm{ID}) / 2$, height $h$, and depth $\delta \theta$ where $\theta$ is small. The cross sectional area, $A$ is defined as $A=h w$ which will consist of an area damaged by the punching process, $A_{d}$ and an undamaged area, $A_{u}$ as shown in Fig. 2. The damaged area is defined to be an area of the sample with a reduced permeability. The value $d$ refers to the length of cumulative damaged width from both edges. The flux is assumed to travel perpendicular to the slice plane.

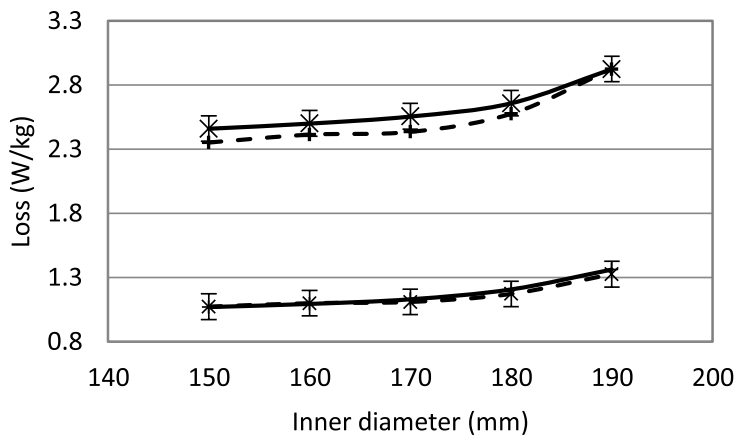

Fig. 3. Comparison of measured (solid lines) and predicted (dashed lines) loses for M250-35A at $50 \mathrm{~Hz}$ and flux densities $1 \mathrm{~T}$ (bottom) and $1.5 \mathrm{~T}$ (top).

The flux passing through the sample, $\phi_{w}$, defined in (1) consists of the flux through the damaged area, $\phi_{d}$, the flux through the undamaged area, $\phi_{u}$, and the flux density, $B_{w}$, similarly defined in (2)

$$
\begin{aligned}
& \phi_{w}=\phi_{d}+\phi_{u} \\
& B_{w}=\frac{d B_{d}+(w-d) B_{u}}{w} .
\end{aligned}
$$

The total power loss, $P_{w}$, is considered to be the sum of the power loss through the damaged region, $P_{d}$, and the power loss through the undamaged region, $P_{u}$

$$
P_{w}=P_{d}+P_{u} .
$$

The magnetically damaged area is defined as having a lower permeability than the rest of the sample. In this simple model, $A_{d}$ is assumed to be damaged to such an extent as to have a permeability no greater than that of a free space and all of the flux travels through $A_{u},(2)$ reduces to

$$
B_{u}=\frac{w}{w-d} B_{w} .
$$

Although a three term power loss model would be more accurate for minimizing the calculation time, it is assumed that the power loss is proportional to the square of the flux density passing through that region, i.e., $P_{u} \propto B_{u}^{2}$ and $P_{w}=P_{u}$ lead to the following equations:

$$
\begin{aligned}
P_{u} & =k_{u} B_{u}^{2} \\
P_{w} & =k_{u}\left[\frac{w}{(w-d)}\right]^{2} B_{w}^{2} .
\end{aligned}
$$

The calculated values for the power loss were compared with the measured values. The extent of the damaged region from the cut edge and the proportionally constant were selected to minimize the difference between the measured and calculated result. This is calculated as a simple percentage difference and was within 5\% for most cases. The values of $d$ and $k_{u}$ were calculated to minimize the cumulative error using the built in solver in Microsoft excel.

\section{RESULTS}

The comparison between the predicted and measured results at $50 \mathrm{~Hz}$ for a range of flux densities are shown in Fig. 3 . The mean parameters obtained by optimizing (6) to produce 
TABLE II

DAMAGED AREA TOTAL LENGTH

\begin{tabular}{ccccc}
\hline \hline ID $(\mathrm{mm})$ & M250 & Std & M330 & Std \\
\hline 150 & 0.603 & 0.019 & 0.639 & 0.011 \\
160 & 0.606 & 0.023 & 0.644 & 0.009 \\
170 & 0.602 & 0.023 & 0.642 & 0.011 \\
180 & 0.599 & 0.032 & 0.648 & 0.019 \\
190 & 0.602 & 0.057 & 0.657 & 0.030 \\
& & & & 0.011 \\
Average & 0.602 & 0.029 & 0.646 & 0.03 different diameter and grade ring samples
\end{tabular}

TABLE III

DAMAGED AREA LENGTH

\begin{tabular}{cccc}
\hline \hline ID $(\mathrm{mm})$ & $\begin{array}{c}\text { daverage } \\
(\mathrm{mm})\end{array}$ & $\begin{array}{c}\text { dinner } \\
(\mathrm{mm})\end{array}$ & $\begin{array}{c}\text { douter } \\
(\mathrm{mm})\end{array}$ \\
\hline M250 & 0.602 & 0.326 & 0.281 \\
M330 & 0.646 & 0.352 & 0.308 \\
Damaged area length for inner and outer edge as a ratio of the cut edge lengths
\end{tabular}

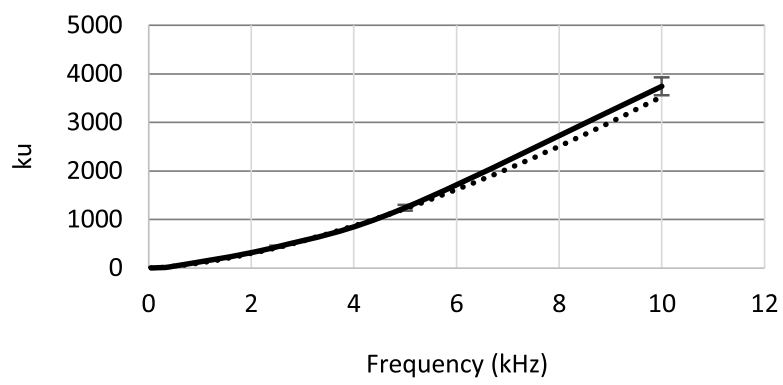

Fig. 4. Variation of $k_{u}$ averaged over the different diameters with frequency for M330 (black line). $k_{u}=0.0056 f^{1.36}$ (dotted line). Error bars $5 \%$.

the lowest cumulative error at the different values of $B_{w}$ for the five internal diameters.

The mean value and standard deviation of $d$ at different frequencies are given in Table II. Assuming that the stress is distributed equally between the two edges, this results in the damaged region extending 0.301 and $0.323 \mathrm{~mm}$ into the M250-35A and M330-35A samples, respectively. However, assuming that the cutting force applied is constant and the stress is proportional to the length of the cut edge, there would exist a larger damaged region at the inner edge, summarized in Table III.

The average values for $k_{u}$ are shown in Fig. 4. This is approximately proportional to the frequency raised to the power 1.36 to within $5 \%$ up to $400 \mathrm{~Hz}$.

It is shown in Fig. 5 that there is a linear relationship between the $k_{u}$ parameter and the area of the cut face per unit mass.

The results show that the power loss increases slightly with decrease in the width. A sharper increase is noted above $170 \mathrm{~mm}$ ID, where the width decreases below $15 \mathrm{~mm}$, as shown in Fig. 6; this could be due to the stress fields and increased dislocation density generated by each cut edges starting to overlap in the center of the sample.

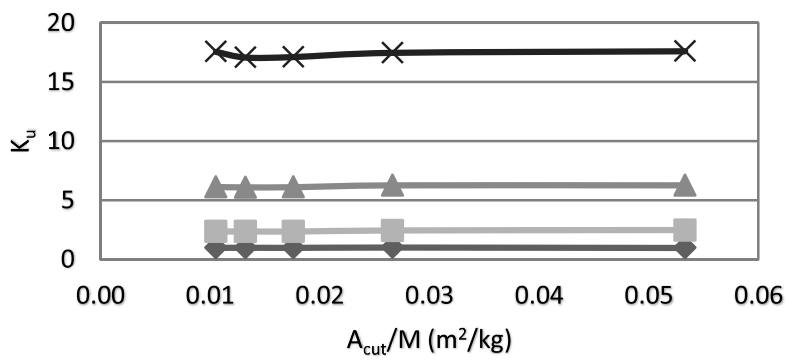

Fig. 5. Ku as a function of cut area per unit mass for the M250-35A sample at frequencies $50 \mathrm{~Hz}$ (bottom), 100, 200, and $400 \mathrm{~Hz}$ (top).

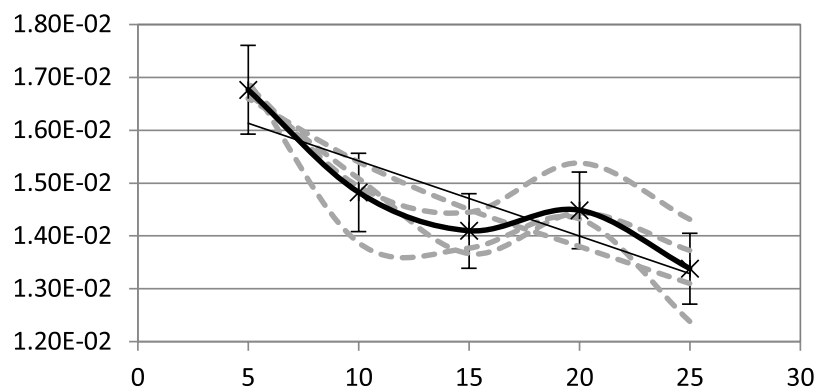

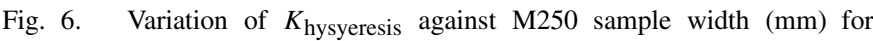
flux densities 1, 1.3, 1.5, 1.7 $\mathrm{T}$ (dashed lines) and average (solid line) with $5 \%$ error bars.

\section{DISCUSSION}

The damaged width does increase slightly as the sample width decreases with a larger increase evident for the $190 \mathrm{~mm}$ M330 samples. This is not unexpected. Although the damaged width being caused by shear stresses during the punching process is independent of frequency, it would play a larger role in the area available for the flux to travel through at high frequencies. This may be what is indicated here. This would imply that the assumption that the flux only travels through the undamaged region would only be valid, where $w \gg d$.

Revisiting (6), we can amend it based on a numerical analysis of the data. The square of the ratio of the total width to undamaged width is approximated as a function of the total width, $w$. The parameter $k_{u}$ is best described as a function of frequency, and the power to which the frequency is raised is consistent for both samples. The constants can be combined to produce a single material dependant property, $K$. Equation (7) gives an approximation for the power loss up to a frequency of $400 \mathrm{~Hz}$ with an average error of $6.23 \%$

$$
\begin{aligned}
P_{w} & \approx K w^{-0.15} f^{1.36} B_{w}^{2} \\
K & =\left\{\begin{array}{ll}
7.09 \times 10^{-3}, & \mathrm{M} 250 \\
9.23 \times 10^{-3}, & \mathrm{M} 330 .
\end{array} \text { for } f \leq 400 \mathrm{~Hz}\right.
\end{aligned}
$$

For higher frequencies above $400 \mathrm{~Hz}$, the power loss is best described using (8), which has an average error of $6.69 \%$

$$
\begin{aligned}
P_{w} & \approx K w^{-0.15} f^{1.54} B_{w}^{2} \\
K & =\left\{\begin{array}{ll}
3.30 \times 10^{-3}, & \text { M250 } \\
\& 3.96 \times 10^{-3}, & \text { M330. }
\end{array} \text { for } f>400 \mathrm{~Hz}\right.
\end{aligned}
$$

The M330 has less deviation from the mean compared with the M250 sample with both showing increased deviation as the sample width decreased with a noticeable jump in both 
cases for the $190 \mathrm{~mm}$ ID rings. There is a consistency across the frequency range.

The damaged widths for the M330 samples are on average $6.75 \%$ greater than their respective M250 samples. This can be explained by increased plastic deformation in the M330 sample The higher Si content of the M250 grade and resulting increase in point dislocations act as hardening agents, which disrupting the movement of the line dislocations through the material, increasing its hardness, reducing the amount of plastic deformation caused, and resulting in a smaller damaged width.

\section{CONCLUSION}

A model to predict power loss as a function of damaged area width is proposed. The power loss increases as damaged width decreases. This is due to a higher dislocation density throughout the sample as a result of the damaged region occupying a larger proportion of the sample.

\section{ACKNOWLEDGMENT}

This work was supported in part by the EPSRC under Grant EP/L504749/1 and by Tata steel, with materials supplied by Cogent Power. The authors would like to thank P. Klimczyk for the experimental work and A. Moses who provided valuable comments.

\section{REFERENCES}

[1] A. Schoppa, J. Schneider, and J.-O. Roth, "Influence of the cutting process on the magnetic properties of non-oriented electrical steels," J. Magn. Magn. Mater., vols. 215-216, pp. 100-102, Jun. 2000.

[2] A. Schoppa, J. Schneider, and C.-D. Wuppermann, "Influence of the manufacturing process on the magnetic properties of non-oriented electrical steels," J. Magn. Magn. Mater., vols. 215-216, pp. 74-78, Jun. 2000
[3] R. Rygal, A. J. Moses, N. Derebasi, J. Schneider, and A. Schoppa, "Influence of cutting stress on magnetic field and flux density distribution in non-oriented electrical steels," J. Magn. Magn. Mater., vols. 215-216, pp. 687-689, Jun. 2000.

[4] K. Senda, M. Ishida, Y. Nakasu, and M. Yagi, "Influence of shearing process on domain structure and magnetic properties of non-oriented electrical steel," J. Magn. Magn. Mater., vol. 304, no. 2, pp. e513-e515, 2006.

[5] V. Maurel, F. Ossart, and R. Billardon, "Residual stresses in punched laminations: Phenomenological analysis and influence on the magnetic behavior of electrical steels," J. Appl. Phys., vol. 93, no. 10, pp. 7106-7108, 2003.

[6] A. J. Moses, N. Derebasi, G. Loisos, and A. Schoppa, "Aspects of the cut-edge effect stress on the power loss and flux density distribution in electrical steel sheets," J. Magn. Magn. Mater., vols. 215-216, pp. 690-692, Jun. 2000.

[7] M. Emura, F. J. G. Landgraf, W. Ross, and J. R. Barreta, "The influence of cutting technique on the magnetic properties of electrical steels," J. Magn. Magn. Mater, vols. 254-255, pp. 358-360, Jan. 2003.

[8] K. H. Schmidt, "Influence of punching on the magnetic properties of electric steel with $1 \%$ silicon," J. Magn. Magn. Mater, vol. 2, nos. 1-3, pp. 136-150, Feb. 1976.

[9] S.-K. Kuo, W.-C. Lee, S.-Y. Lin, and C.-Y. Lu, "The influence of cutting edge deformations on magnetic performance degradation of electrical steel," in Proc. 17th Int. Conf. Electr. Mach. Syst. (ICEMS), Oct. 2014, pp. 3041-3046.

[10] G. Loisos and A. J. Moses, "Effect of mechanical and Nd:YAG laser cutting on magnetic flux distribution near the cut edge of nonoriented steels," J. Mater. Process. Technol., vol. 161, pp. 151-155, Apr. 2005.

[11] T. Nakata, M. Nakano, and K. Kawahara, "Effects of stress due to cutting on magnetic characteristics of silicon steel," IEEE Transl. J. Magn. Jpn., vol. 7, no. 6, pp. 453-457, Jun. 1992.

[12] Z. Gmyrek, A. Cavagnino, and L. Ferraris, "Estimation of magnetic properties and damaged area width due to punching process: Modeling and experimental research," in Proc. 20th Int. Conf. Electr. Mach. (ICEM), Sep. 2012, pp. 1301-1308.

[13] S. Zurek, P. Marketos, T. Meydan, and A. J. Moses, "Use of novel adaptive digital feedback for magnetic measurements under controlled magnetizing conditions," IEEE Trans. Magn., vol. 41, no. 11, pp. 4242-4249, Nov. 2005. 